\title{
El perdón: condición para la paz. Estudiantes de Salud Pública y Enfermería, Universidad de Antioquia
}

\author{
The forgiveness: a condition for the peace. Nursing and Public Health \\ students University of Antioquia
}

\author{
Álvaro Giraldo Pineda, Universidad de Antioquia \\ alvaro.giraldo@udea.edu.co | http://orcid.org/0000-0001-8942-6750 \\ Calle 67 No. 53-108. Medellín-Antioquia \\ Constanza Forero Pulido, Universidad de Antioquia \\ constanza.forero@udea.edu.co | http://orcid.org/0000-0001-7494-6825 \\ Johana Meliza Córdoba Ibargüen, Universidad de Antioquia \\ meliza.cordoba@udea.edu.co | http://orcid.org/0000-0002-6822-7821 \\ Anghie Phamela López Mejía, Universidad de Antioquia \\ lopez@udea.edu.co | http://orcid.org/0000-0003-4385-6290 \\ Gloria Estella Estrada Bedoya, Universidad de Antioquia \\ gestrada0622@gmail.com | http://orcid.org/0000-0002-3054-7141 \\ Teresa Mutumbajoy Tandioy, Universidad de Antioquia \\ killatere@gmail.com | http://orcid.org/0000-0001-7171-1246 \\ Miguel Ángel Orrego Rodríguez, Universidad de Antioquia \\ miguel.orrego@udea.edu.co | http://orcid.org/0000-0002-5908-054
}

\begin{abstract}
Resumen
Colombia está viviendo un momento histórico con la firma de los acuerdos de paz que dan fin a una violencia de más de 50 años. Importa saber qué significa para las personas la paz, en especial para los estudiantes de Salud Pública y Enfermería quienes son parte del futuro y la transformación del país. Objetivo: comprender el significado que le dan a la paz los estudiantes de Salud Pública y Enfermería. Metodología: investigación cualitativa - etnográfica, realizada en Medellín, Colombia. Se entrevistaron 16 estudiantes. Para el análisis de la información se
\end{abstract}


codificaron y categorizaron las entrevistas. Ética: El principio de confidencialidad y el respeto mutuo fueron los criterios que primaron durante la investigación. Resultados: Los estudiantes consideran la paz como derecho y deber, por lo tanto tienen que comprender a las personas que han participado en el conflicto armado en Colombia, para lograr tranquilidad en la población; para algunos la paz significa un imposible, para otros puede ser viable. El perdón es ambiguo porque está condicionado a los sentimientos que les generó el conflicto armado; los estudiantes que tuvieron experiencias directas perdonan, si los excombatientes sufren y los de experiencias indirectas, perdonan si estos pagan por los delitos. Conclusión: Hay dos miradas sobre la paz y una es que la paz es un imposible, por lo tanto ni entienden ni perdonan; la otra mirada es que la paz es posible y se perdona.

\begin{abstract}
Colombia is experiencing a historic moment after the signing of the peace agreements that ended over 50 years of violence. It is important to know the meaning of peace for people, especially for nursing and Public Health students because they are part of the future and the transformation of the country. Objective: to understand the meaning that Nursing and Public Health students give to peace. Methodology: qualitative - ethnographic research, carried out in Medellín, Colombia. 16 students were interviewed. For the analysis of the information, the interviews were codified and categorized. Ethics: Confidentiality and mutual respect were the criteria that prevailed during the investigation. Results: Students consider peace as a right and a duty. To achieve tranquility in the population it is necessary to understand the people who have been part in the armed conflict in Colombia. For some people peace means an impossible, for others it can be viable. Forgiveness is ambiguous because it is conditioned by the feelings generated during the armed conflict; for the students that report direct experiences forgiveness is conditioned to the suffering of the excombatants, and for those with indirect experiences, forgiveness can happened if they pay for the crimes. Conclusion: There are two views about peace, for some students peace is impossible, therefore people do not understand and they will not forgive; for others peace is possible, and people can forgive.
\end{abstract}

Palabras clave: conflicto, paz, etnografía, perdón, entrevistas.

Keywords: conflic, peace, ethnography, forgiveness, interviews.

\title{
1. INTRODUCCIÓN
}

El conflicto colombiano lleva más de 50 años y ha dado lugar a diferentes procesos de paz como los realizados en 1986 y 1992 con grupos como el M-19, el Partido Revolucionario de los Trabajadores PRT, el Ejército Popular de Liberación EPL y el Quintín Lame (Zapata, 2009). Los diálogos de paz más recientes en Colombia son los del gobierno del presidente Juan Manuel Santos y las FARC - EP, que comenzaron en Oslo, Noruega, continuaron en la Habana, Cuba y culminaron con la firma del Acuerdo de paz para la terminación definitiva del Conflicto armado en Colombia (Gobierno Nacional de Colombia y Fuerzas Armadas Revolucionarias de Colombia 
Ejército del Pueblo, 2016). El acuerdo inicial lo firmaron en Cartagena de Indias las partes involucradas y debía refrendarse con un plebiscito. El resultado final concluyó con la victoria del "No", lo cual obligó al gobierno, en cabeza del presidente Juan Manuel Santos, a "renegociar" el acuerdo, tomando en consideración las objeciones de los opositores; esto generó inseguridad sobre la aplicabilidad legal de dichos acuerdos.

Transcurrido un período de negociación entre el gobierno y los opositores, se firmó un nuevo texto entre las FARC - EP y el Gobierno, el 24 de noviembre de 2016 en el Teatro Colón de la ciudad de Bogotá, Colombia, el cual fue ratificado por el Senado de la República y la Cámara de Representantes los días 29 y 30 de noviembre de 2016 (Salcedo, 2016).

La firma de estos acuerdos genera un compromiso de todos los colombianos en la construcción de una cultura de paz, de la cual la Universidad, especialmente la pública, no puede estar ajena. Esta debe tener una visión de lo que los futuros profesionales de la salud piensan acerca de la paz partiendo de sus experiencias, tanto directas como indirectas y del papel de los medios de información en pro de la búsqueda de la paz.

Se pueden encontrar diversas connotaciones sobre la paz, por ejemplo Galtung (2003), habla sobre la paz positiva que va más allá de la ausencia de violencia directa y la presencia de justicia social, y paz negativa entendida como la ausencia de guerra o conflictos destructivos. Por su parte Lederach (2000), plantea que para pasar de una paz negativa a una positiva, se requieren igualdad y reciprocidad en las interacciones. García y Carrillo (2017), encontraron que para los estudiantes universitarios, es posible crear un ambiente de paz estable y duradera en todo el territorio colombiano.

La universidad pública debe reflexionar sobre sí misma y otros aspectos como: las transformaciones políticas y culturales que vive el país, la interpretación de las realidades sociales y su papel como mediadora en las relaciones entre el Estado y la sociedad (De Sousa, 2007). La Universidad de Antioquia tiene diferentes programas que pertenecen a diversas áreas del conocimiento, cada una con su propósito y su compromiso social. Las Facultades de Salud Pública y Enfermería forman estudiantes de todos los sectores sociales, especialmente estratos uno, dos y tres, quienes provienen de diferentes regiones del país, tanto urbanas como rurales, con distintas experiencias en relación con el conflicto armado, que les ha permitido la construcción de significados, tanto de la violencia, como de la paz. Basados en ello nos interesa la comprensión de los significados de paz, con el fin de que sirvan de base para la construcción de proyectos educativos, diseños de talleres y cursos que preparen a los estudiantes para ser gestores de paz.

Para lograr lo anterior, esta investigación planteó como objetivo "comprender los significados que le dan a la paz los estudiantes de Salud Pública y Enfermería”. 


\subsection{Marco Referencial}

Para la escuela socrática, la paz es la antítesis de la guerra. Para Aristóteles, la guerra tiene cabida siempre que sea necesaria para alcanzar la Paz. Por su parte, Maquiavelo la cataloga como la ausencia de guerra. Kelsen expresa que "es una situación que se caracteriza por la ausencia de la fuerza" y presenta el Derecho como un orden para promover la paz, que prohíbe a los miembros de una comunidad el uso de la fuerza (Moreno, 2014).

El Artículo 22 de la Constitución Política de Colombia de 1991 concibe la paz como un derecho y un deber de obligatorio cumplimiento. La Corte Constitucional de Colombia define la paz como ausencia de conflictos o enfrentamientos violentos y la asume como estado ideal desde la perspectiva de derecho fundamental y derecho colectivo, que puede ser objeto de protección, incluso mediante la acción de tutela. Además, está consignada en diversos tratados sobre derechos humanos, de los cuales Colombia es parte.

Muñoz y otros (2001), describen la paz como un signo de bienestar, felicidad y armonía con el universo en su conjunto y es lo que le da sentido a nuestras vidas, además indica que la paz permite darle salidas satisfactorias a los conflictos y aunque hoy en día se nos hace difícil identificarla, es sin duda una de las grandes preocupaciones en todos los ámbitos que rodean al ser humano. "Son todas aquellas realidades en las que se regulan pacíficamente los conflictos, en las que se satisfacen al máximo las necesidades y los objetivos de los actores implicados, sean cuales sean estos, o los momentos o los espacios donde se producen".

Según Martínez (2007), hay suficiente demanda de paz y lo que se necesita es ofrecer alternativas para la resolución de conflictos y considera que la paz crece como un proceso casi inconsciente de aprendizaje.

\section{METODOLOGÍA}

Para desarrollar el objetivo planteado, se utilizó la investigación cualitativa, cuyas características son: comprensividad de los fenómenos, capacidad de estudiarlos dentro de un contexto específico como totalidades; flexibilidad e interés por captar la mirada de los participantes (Patton, 1990).

También se caracteriza por ser inductiva, ya que las categorías de análisis emergen durante la recolección de la información y permite identificar e interpretar los significados para encontrarle sentido a una problemática que presenta una comunidad o población.

El enfoque es etnográfico, definido por Boyle (2003) como descripción e interpretación de un grupo social o cultural, que involucra las miradas emic (desde los participantes) y etic (desde los investigadores), lo que permite una aproximación a la población. Como el objetivo de este trabajo es comprender experiencias y significados, esos dos elementos constituyen una visión de la cultura desde lo histórico hermenéutico o comprensivo y por lo tanto se parte de la definición de cultura de Geertz (2003), que la entiende como "estructuras de significación socialmente 
establecidas". La etnografía depende de la mirada de los actores mismos que viven los procesos: "mirada emic", sin desconocer los aportes teóricos y conceptuales del investigador (Geertz, 2003)..

La población estuvo compuesta por hombres y mujeres, estudiantes de pregrado de Salud Pública y Enfermería, que hubiesen cursado el tercer semestre o más y que accedieron voluntariamente a formar parte de la investigación. El procedimiento para lograr la participación de los estudiantes se hizo por medio de la difusión del proyecto en cada una de las Facultades, solicitando voluntarios para participar en el proyecto; se utilizó la técnica de bola de nieve con el fin de obtener más participantes y se solicitó a los que respondieron las entrevistas que sugirieran nombres de compañeros y compañeras que pudieran participar brindando información.

\subsection{Técnicas}

La Entrevista: el uso de la entrevista fue fundamental en este estudio, para obtener de los participantes información sobre el significado que le dan a la paz. Se realizaron dieciséis (16) entrevistas abiertas, con una duración aproximada de 45 minutos cada una, estas fueron grabadas con previa aceptación de los participantes y antes de comenzar se destinó un espacio para que el entrevistado leyera el consentimiento informado y realizara las preguntas que considerara necesarias sobre la investigación; con este documento firmado, se procedió a iniciar la entrevista

Las entrevistas fueron realizadas por los investigadores y se llevaron a cabo en el lugar seleccionado para tal fin, se tuvo en cuenta no llevar preguntas específicas sino una lista de temas y se inició con preguntas amplias como: "hábleme de...", "cuénteme sobre..." y los temas fueron paz, sentimientos, experiencias directas e indirectas. Una condición importante fue el contacto previo con los participantes, de tal manera que cuando se realizó la entrevista había confianza y claridad sobre el objetivo, los contenidos y los compromisos, tanto de los participantes como de los investigadores. Las transcripciones se guardaron en el archivo de la línea de Investigación Cultura y Salud, utilizando seudónimos.

Se elaboró el diario de campo, en el que se registraron encuentros con los participantes, comentarios y demás aspectos que ayudaron a la comprensión del fenómeno, tales como: sentimientos de los investigadores, conceptos sobre determinadas situaciones, reflexiones metodológicas y descripción de los encuentros con los participantes.

\subsection{Análisis de la información}

El análisis de la información se desarrolló de la siguiente manera: una vez realizada la primera entrevista se transcribió y se entregó una copia a cada uno de los integrantes del grupo para hacer una lectura y escribir comentarios generales sobre elementos formales y de contenido; se fijó una fecha para una nueva reunión en la cual cada integrante expondría los comentarios.

Posteriormente se hizo una lectura grupal de la entrevista para separar unidades de sentido e identificar los códigos; estas unidades se recortaron y se pegaron en una hoja de papel con el 
nombre del código correspondiente, así se realizó la codificación como proceso de caracterizar y clasificar los datos (Velasco \& Díaz de Rada, 2004). Se agruparon los códigos similares, identificando las categorías y subcategorías para diseñar los mapas conceptuales, que facilitaron la construcción del informe final, el cual fue complementado con la revisión bibliográfica que permitió su confrontación con los datos. La categoría principal fue "la paz" y las subcategorías: "experiencia directa", "experiencia indirecta" y "el perdón" como categoría emergente.

\subsection{Criterios de rigor}

Las entrevistas fueron realizadas por dos entrevistadores: uno principal que se encargó de llevar a cabo toda la entrevista y uno secundario que apoyó y complementó la información. La transcripción se hizo en los dos días siguientes a su realización, con el fin de lograr un alto grado de fidelidad (Castillo \& Vásquez, 2003), posteriormente las entrevistas se discutieron con otros investigadores.

El análisis de la información se realizó en el grupo de investigación lo que permitió discutir y enfatizar la mirada de los participantes; de esta manera se proporcionó el espacio adecuado para extraer de cada entrevista el máximo de información según los objetivos del estudio. La recolección de la información se suspendió cuando hubo saturación de la información, es decir, cuando las unidades de sentido en las que se separa cada entrevista, se repitieron.

\subsection{Consideraciones éticas}

El principio de confidencialidad y el respeto mutuo fueron los criterios que primaron durante la investigación. La inclusión de criterios éticos se realizó desde el diseño y presentación de la propuesta de investigación, teniendo en cuenta desde este primer momento, los riesgos que pudieran presentarse.

Según la resolución 8430 (República de Colombia, Ministerio de Salud, 1993), que regula la investigación con seres humanos en Colombia, esta investigación se clasifica como de riesgo mínimo, ya que no implica ninguna intervención o modificación sobre la vida o las condiciones emocionales de los participantes. Durante el desarrollo de la investigación los participantes estuvieron en plena libertad de no responder preguntas o retirarse de la investigación.

Cabe aclarar que durante las entrevistas no hubo negativas, ni se presentaron dificultades que impidieran su realización. Los participantes pudieron expresarse de manera libre. Algunos de ellos, al final de la entrevista, se mostraron agradecidos por el hecho de que alguien los escuchara y les permitiera expresar esos sentimientos que tenían reprimidos y que no habían podido manifestárselos a alguien.

Esta investigación fue aprobada por el Comité de Ética de la Facultad Nacional de Salud Pública de la Universidad de Antioquia mediante el acta $N^{\circ}$ 2016-13100 de 2017. 


\section{RESULTADOS Y DISCUSIÓN}

Para los estudiantes de las Facultades de Salud Pública y Enfermería de la Universidad de Antioquia, la Paz es un derecho y un deber, tal como lo plantea la Constitución Colombiana (Asamblea Nacional Constituyente, 1991). También es la resolución de un conflicto armado que ha venido afectando la sociedad civil y del cual los estudiantes tienen un concepto negativo.

La paz es un derecho, es un deber. El conflicto es una maldición, la maldición generacional E1

El proceso de paz realizado en Colombia, inicia con la firma e implementación de los acuerdos entre las FARC - EP y el Gobierno, con los cuales se espera que disminuya la violencia, se restaure el bienestar de la población y la vida en el campo. Es lo que Galtung (2003) denomina paz negativa y que la entiende como "la ausencia de enfrentamientos violentos y mecanismos para alcanzar la solución de los conflictos existentes".

Las consecuencias, pueden ser positivas, porque si todos nos acoplamos a este acuerdo, tal vez no se consiga como tal la paz, como todos queremos, pero al menos se logre mitigar o disminuir un poquito la guerra, la violencia E3

Según la ley 1448 de 2011 (Congreso de la República de Colombia, 2011), en Colombia se considera víctima a toda persona que haya sufrido daños, individual o colectivamente, incluidas lesiones físicas o mentales, sufrimiento emocional, pérdidas económicas o menoscabo sustancial de sus derechos fundamentales, como consecuencia de acciones u omisiones que constituyan una violación manifiesta de las normas internacionales de derechos humanos o una violación grave del derecho Internacional humanitario. Los participantes se consideran víctimas del conflicto armado, al igual que lo es toda la población, debido a que el país en general ha sido desangrado durante más de cincuenta años. Las personas que por sus condiciones económicas y sociales se vieron obligadas a empuñar un fusil o fueron reclutadas en contra de su voluntad, también se consideran víctimas.

Yo creo que en sí todos hemos sido víctimas, algunos muy directamente y otros de manera indirecta E13

Para lograr la paz algunos estudiantes consideran que cada persona debe comprender a los que han participado en el conflicto y garantizar la confianza, condicionada a lo que el gobierno les ofrece a los excombatientes y de esta manera lograr la tranquilidad de la población colombiana. Otros estudiantes plantean que obtener la paz es casi imposible porque la gente no cambia su forma de ser ni su comportamiento, no hay confianza, no hay justicia, desconociendo el planteamiento de Kant sobre la confianza como elemento fundamental para obtener la paz. (Kant, 1795).

El que es, no deja de ser E9

Ámbitos. Revista Internacional de Comunicación | ISSN: 1139-1979 | E-ISSN: 1988-5733, Nº. 44 (2019) 
Los excombatientes deben tener compromiso con el estudio, la salud, aprovechar las oportunidades que les brinda el gobierno e información a la población de ese compromiso E1

Los estudiantes de Enfermería y de Salud Pública, expresan en las entrevistas la necesidad del perdón para construir una Colombia en paz, que requiere cambios, y con rencores no es posible construirla. Este perdón es ambiguo y depende del tipo de experiencias, indirectas o directas, que hayan tenido los estudiantes y que estén relacionadas con el conflicto armado.

Se puede superar de alguna manera y perdonar a sus victimarios y construir una paz, o una sociedad justa y equitativa E13.

\subsection{Experiencias indirectas}

Se denomina experiencia indirecta, aquella que los participantes han conocido por medio de sus familiares, medios de información y compañeros.

Los relatos de los familiares acerca del conflicto armado no son considerados como elementos cercanos, porque no fueron presenciados por ellos mismos, sino por algún miembro de su grupo familiar, sin embargo, son acogidos como aprendizaje, porque expresan vivencias confiables, aunque prefieren no profundizar mucho sobre ellas, porque son dolorosas y reviven los sentimientos generados por aquella experiencia.

Mi familia casi nunca toca ese tema, inclusive con lo que le pasó a mis tíos, pues uno se vino a dar cuenta ya muy grande, es que a uno chiquitico no le contaban E 2

Para los estudiantes los medios de información como la radio, la prensa y la televisión, son fuentes consideradas no confiables, sesgadas según el interés particular que tenga cada medio, lo cual no les permite tener una visión cercana a la realidad. Esta situación concuerda con el estudio de Urbina (2016), quien describe la utilización de todo tipo de estrategias para difamar y desinformar acerca de los acuerdos alcanzados en la agenda que se discutió en La Habana.

Los medios siempre los he visto como muy sesgados, porque yo lo viví en un conflicto y eso no aparecía en los medios, entonces uno dice: ¿esto a qué obedece que no está en el camino de nuestra realidad? E 4

Los estudiantes también obtienen información mediante la discusión y los momentos de reflexión con los compañeros, muchas veces a partir de la experiencia o desde lo relatado por los medios de información. Estas discusiones son consideradas como incompletas y de bajo nivel de profundidad, porque no reciben respaldo de la universidad, y en general los docentes no les brindan orientación para realizarlas. Al respecto, en el estudio de Iguarán, Forero y Velandia (2014)los docentes confirman lo dicho por los participantes de este estudio, reconociendo que debido a dificultades como la carga académica y laboral y la orientación de la Universidad, que exige que se traten estrictamente los contenidos específicos de cada disciplina, no permiten que se aborden estos temas adecuadamente, y no favorecen la discusión ni la reflexión. 
Son discusiones que se dan frente al grupo de compañeros, pero no avaladas bajo algo, por una razón, por el estudio o por un docente E1

Otro medio de información según los participantes es la iglesia, que pretende orientar, pero que en algunos casos da información que no es verídica, porque responde más a sentimientos u opiniones personales. Según Restrepo y Ramírez (2016), las iglesias influenciaron el voto por el No en el plebiscito, que puso en crisis los acuerdos de paz.

La iglesia nos decía que cómo vamos ayudar a personas que lo único que hacen es pensar en ellos y no en Dios E9

Aunque los estudiantes que tuvieron experiencias indirectas reconocen tener poco conocimiento acerca del conflicto armado, expresan sentir miedo ante la posibilidad de que alguna de estas situaciones pueda ocurrirles a ellos. Este tipo de miedo, resultante de relatos de experiencias de otras personas, corresponde a la definición de un trastorno de ansiedad, según lo plantean Hewitt y otros (2016), en el estudio realizado con adultos expuestos al conflicto armado en Colombia.

Miedo a sufrir lo que otros han vivido o nos han contado. E2

Los participantes consideran que deben superar el miedo y lograr confianza para perdonar a los excombatientes y estos deben cumplir un proceso de resocialización, el cual incluye el juzgamiento y el pago de los delitos que cometieron y así lograr que se reincorporen a la vida civil y puedan vivir como todo el mundo E4. lo que concuerda con el trabajo de Castellanos \& Nicolás (2016), quienes señalan que los mecanismos de justicia, aplicados a los actores armados en el proceso de paz, son definitivos para incrementar la confianza de la población colombiana hacia el perdón y la reconciliación nacional. Estas condiciones deben ser proporcionadas por la sociedad, evitando que los excombatientes reincidan en actividades ilegales, lo que se articula con el concepto de perdón planteado por Byrom (1999), quien expresa que este ha dejado de ser concebido como religioso, para convertirse en algo que finalmente la sociedad ha entendido como un acto para romper el ciclo de la violencia.

Esto implica tener un trabajo y una familia, llegar a su casa, estar dentro de un tejido social, sentirse parte de una comunidad E4.

Que paguen, que paguen con cárcel para ser perdonados. E8

\subsection{Experiencia Directa}

Se denomina experiencia directa, aquella que los participantes han vivenciado solos o junto a sus familiares en las diferentes zonas en las que se ha presentado el conflicto armado. Los estudiantes que tuvieron experiencias directas consideran que el conflicto armado marcó sus vidas, por el impacto de la violencia y porque se vieron amenazados. 
Éramos en la cancha jugando y de momento llegaba la guerrilla, las FARC, las AUC, entonces todo el mundo salía corriendo de la cancha E7

Nos tocó ver morir a muchas personas cercanas E8

Lo amenazaban a uno y uno sin tener nada que ver E8

Frente a estas situaciones, los afectados debieron buscar estrategias para sobrevivir como esconderse o abandonar su territorio, para no tener que vivir bajo presión permanente o no entrar en conflicto con los distintos grupos armados.

No vamos a ser sus enemigos, o sea, si usted viene nos pide un favor, comida, agua, normal aquí se la vamos a dar E 1

Alejarse, primero por mi propia seguridad física y porque uno puede perder la vida E4

Nosotros quedábamos en el medio como de las balas cruzadas, me tocó, pues meternos con toda mi familia debajo de las camas para protegernos. E2

Estas estrategias coinciden con lo encontrado por López y Rodríguez, quienes además plantean el distanciamiento físico como la forma utilizada para sobrevivir, este abandono en algunos casos produjo la desintegración temporal de las familias. (López \& Rodríguez, 2012).

Mis abuelos tuvieron que dejar sus tierras, porque el conflicto armado hizo como presencia, entonces ellos más que todo por miedo, deciden venirse para la ciudad E16

El miedo es el sentimiento predominante debido a las experiencias pasadas y se revive ante algunas situaciones de la cotidianidad que les hace recordar esos momentos y por esta razón evitan estar presentes en sitios que consideran peligrosos, esto coincide con el estudio planteado por Andrade (2011).

Veo una calle muy sola y digamos voy para la casa, tipo once y media, las calles están muy solas y la verdad siento como delirio de persecución, como que alguien me está mirando. Sí, he sentido miedo, esos momentos me hacen recordar mucho. E7

También evitan pasar por sitios donde fueron asesinadas personas por temor a que estas hagan su aparición y los asusten y si lo tienen que hacer, buscan compañía para no pasar solos.

Me daba mucho miedo que me iba a aparecer un fantasma y como esa sangre no la lavan, ni nada, y uno pasa y ve la sangre ahí, yo vi donde la mataron y uno queda sabiendo donde, entonces por ahí no paso. E11

Otros de los sentimientos que tuvieron durante el conflicto armado, fueron rabia y rencor al ver la angustia de sus familiares que se debieron desplazar a otros sitios, algunos quedaron en 
medio del fuego cruzado y otros se sintieron impotentes frente a la fuerza de los agresores. Además, los estudiantes manifestaron su frustración por no ver soluciones para terminar con los enfrentamientos y tristeza por los excombatientes, al ver que algunos eran conocidos, amigos de infancia o familiares, que no razonaban a la hora de actuar, por seguir unos ideales.

Mucho rencor de los que hemos vivido esta experiencia de la guerra, mucha rabia, porque ellos piensan que uno está como ayudando a los otros E6

Tristeza, porque son personas que no deben estar en todos sus cabales, para uno llegar y matar al otro, así tan fríamente, yo siento lástima porque está en lo más bajo como persona E10

Los sentimientos de las víctimas del conflicto armado encontrados por Villa (2016), fueron: rabia, dolor, tristeza, deseo de venganza y resentimiento, que marcan sus vidas de manera permanente, lo que sucede también con los participantes de este estudio.

Los participantes que tuvieron experiencias directas, consideran que para perdonar debe haber justicia equitativa, según los daños ocasionados por los grupos armados y deben cumplir unos compromisos que incluyen: reconocer que cometieron delitos, pagar por ellos, sufrir de la misma manera que hicieron sufrir y que les duela. Lo anterior coincide con los resultados del estudio de Castellanos \& Nicolás (2016), en el que se encontró que como solución al problema de la violencia, se debe aplicar el principio de "ojo por ojo y diente por diente", y de esa manera lograr la paz.

Ellos hicieron sufrir, ¿por qué no sufrir ellos también? yo digo que ellos deberían de pagar. E8

Para finalizar es importante aclarar que independiente del tipo de experiencia, los significados que los participantes dan a las situaciones de conflicto y violencia se refieren, en general, a todos los grupos armados que existen en el territorio nacional.

\section{CONCLUSIONES}

La paz es considerada como derecho y deber, lo que implica comprender a las personas que participaron en el conflicto armado.

Para que haya paz debe haber perdón y este es ambiguo y condicionado, según la experiencia de los participantes. Quienes han tenido experiencias indirectas, opinan que los excombatientes del conflicto armado deben pagar y los que han tenido experiencias directas, opinan que deben sufrir como los hicieron sufrir.

Para los estudiantes la información obtenida por los compañeros, los profesores y los medios (prensa, radio y televisión) en relación con la paz, es poca y no confiable.

El miedo es un sentimiento predominante en los participantes, independiente del tipo de experiencia. 
La utilización de la etnografía comprensiva, posibilitó identificar los significados sobre la paz, logrando de esta manera una mirada emic.

La entrevista, según manifestaron los participantes, les permitió expresar sentimientos guardados durante mucho tiempo.

\subsection{Recomendaciones}

Se debe profundizar en lo referente a los significados de paz en otras poblaciones diferente a los estudiantes de Enfermería y Salud Pública.

Hacer un trabajo en el cual las personas comprometidas con el proceso de paz, conformen grupos para que se realice una pedagogía de la paz y el perdón en los ciudadanos.

Mayor compromiso de las universidades, especialmente las públicas, en apoyar la construcción de la cultura de paz.

Elaborar propuestas para el trabajo dentro de las facultades, fundamentado en el significado que los estudiantes le dan a la paz.

\subsection{Agradecimientos}

A la Universidad de Antioquia por su apoyo para el desarrollo de este estudio.

A los estudiantes de las Facultades de Salud Pública y Enfermería de la Universidad de Antioquia que participaron de manera voluntaria en esta investigación y a todos los integrantes de la Línea de Investigación "Cultura y Salud".

\section{REFERENCIAS}

Andrade, J. (2011). Efectos Psicopatológicos del conflicto armado Colombiano en familias en situación de desplazamiento forzado reasentadas en el municipio del Cairo en el año 2008. Orbis. Revista Científica Ciencias Humanas, VII(20), 111-114.

Asamblea Nacional Constituyente. (13 de 06 de 1991). Constitución Política de Colombia. Ley de la Republica de Colombia. Bogotá, D.C, Colombia .

Boyle, J. (2003). Estilos de etnografía. En J. Morse, Asuntos críticos en los métodos de investigación cualitativa (págs. 185-214). Medellín: Universidad de Antioquia .

Byrom, M. (3 de Mayo de 1999). The Journal of Humanitarian Assistance. Obtenido de Field experience and current research on humanitarian action and policy: https://sites.tufts.edu/jha/archives/140

Castellanos, J., \& Nicolás, D. (14 de Abril de 2016). Repositorio Institucional Universidad Distrital - RIUD, Tesis y Disertaciones Académicas Facultad de Ciencias y Educación, Maestría en Educación. Obtenido de http://repository.udistrital.edu.co/handle/11349/2633 
Castillo, E., \& Vásquez, M. (2003). El rigor metodológico en la investigación cualitativa. XXXIV(3).

Congreso de la República de Colombia. (2011). Ley 1448 de 2011. Ley. Bogotá.

De Sousa, B. (2007). La Universidad en el siglo XXI. Para una reforma democrática y emancipatoria de la universidad. (Septima ed.). Bolivia: Plural editores.

Galtung, J. (2003). Paz por medios pacíficos. Paz y conflicto, desarrollo y civilización. Bilbao: Gemika Gogoratuz.

García, C., \& Carrillo, M. (2017). Significados, obstáculos y formas de Construcción: la paz desde los estudiantes universitarios. Revista Universidad Católica Luis Amigó, 222 - 241.

Geertz, C. (2003). La interpretación de las culturas. Barcelona: Gedisa S.A.

Gobierno Nacional de Colombia y Fuerzas Armadas Revolucionarias de Colombia Ejército del Pueblo. (2016). Acuerdo final para la terminación del conflicto y la construcción de una estable y durdera. Recuperado el 09 de Noviembre de 2017, de Oficina del Alto Comisionado para la Paz: http:// www.altocomisionadoparalapaz.gov.co/procesos-y-conversaciones/Paginas/Texto-completo-delAcuerdo-Final-para-la-Terminacion-del-conflicto.aspx

Hewitt, N., Juárez, F., Parada, A., Guerrero, J., Romero, Y., Salgado, A., \& Vargas, M. (2016). Afectaciones Psicológicas, Estrategias de Afrontamiento y Niveles de Resiliencia de Adultos Expuestos al Conflicto Armado en Colombia. Revista Colombiana de Psicología, XXV(1), 125-140.

Iguarán, N., Forero, D., \& Velandia, M. (2014). Rol del docente universitario frente a la paz y reconciliación en Colombia. Revista de la Universidad de la Salle, I, 135 -147.

Kant, I. (1795). La Paz Perpetua. La fundación el libro total y SIC editorial.

Lederach, J. (2000). El abecé de la paz y los conflictos. Madrid: Catarata.

López, G., \& Rodríguez, A. (2012). El lugar de los sentimientos, el lugar de los vínculos. Reconfiguración de identidades en el marco del conflicto armado colombiano. Revista Colombiana de Ciencias Sociales, III(2), 270-289.

Martínez, J. (2007). KENNETH E. BOULDING, ECONOMISTA Y PACIFISTA. Textos de Economía, Paz y Seguridad, I(1). Obtenido de http://www.eumed.net/rev/tepys/01/jcmc-1.htm

Moreno, F. (2014). El concepto de paz en la constitución política de colombia de 1991 reconstrucción dialéctica de su significado a partir de la jurisprudencia de la corte constitucional. Revista de Derecho Universidad Católica del Norte, 21(2), 305 - 346. Obtenido de http://www.scielo.cl/pdf/ rducn/v21n2/art09.pdf

Muñoz, F., Martínez, V., Fernández, A., Magallón, C., Molina, B., Cano, M., Tortosa, J. (2001). La paz Imperfecta. Granada, España: Universidad de Granada, Colección Eirene.

Patton, M. (1990). Qualitative research and evaluation methods. Beverly Hills: 3 edition Sage Publications. República de Colombia, Ministerio de Salud (1993). Resolución № 008430. Resolución. Bogotá.

Restrepo, L., \& Ramírez, S. (2016) Colombia: sorpresas y sobresaltos de la paz. Revista Nueva Sociedad 266, 129-139. 
Salcedo, C. (2016). Posiciones y riesgos para la paz en Colombia. Revista en línea, Palabras al margen.

Urbina, J. (2016). Jóvenes universitarios en Colombia: entre la desinformación, el pesimismo y los anhelos de paz. Argumentos, XXIX(81), 87 - 107.

Velasco, H., \& Díaz de Rada, A. (2004). La lógica de la investigación etnográfica. Un modelo de trabajo para etnógrafos de escuela. Madrid: 4 ed, Trotta.

Villa, J. (2016). Perdón y reconciliación: una perspectiva psicosocial desde la noviolencia. Polis. Revista Latinoamericana, XV(43), 131-157.

Zapata, M. (2009). Construcción de paz y transformación de conflictos. En M. Bello, Acción sin daño y reflexiones sobre prácticas de paz. Una aproximación desde la experiencia colombiana (págs. 4- 51). Bogotá D.C: Bluegraphic diseño y publicidad. 